\title{
Sports Dance Action Recognition System Oriented to Human Motion Monitoring and Sensing
}

\author{
Shasha Ni $(\mathbb{D})$ and Dawei Yao \\ College of Physical Education, Qiqihar University, Qiqihar, 161006 Heilongjiang, China \\ Correspondence should be addressed to Shasha Ni; nishasha@qqhru.edu.cn
}

Received 11 January 2021; Revised 13 April 2021; Accepted 31 May 2021; Published 14 June 2021

Academic Editor: Wenqing Wu

Copyright (C) 2021 Shasha Ni and Dawei Yao. This is an open access article distributed under the Creative Commons Attribution License, which permits unrestricted use, distribution, and reproduction in any medium, provided the original work is properly cited.

\begin{abstract}
Because of its high research value, action recognition has become a very popular research direction in recent years. However, the research on the combination of motion recognition technology and dance movements is still in its infancy. At the same time, due to the high complexity of dance movements and the problems of human body self-occlusion when performing dances, research on dance video action recognition has been caused. Progress is relatively slow. This article mainly introduces the research of sports dance action recognition system oriented to human motion monitoring and sensing, fully considers the abovementioned problems, and makes in-depth research and analysis on the current excellent action recognition research content in this field. This paper proposes a research method of sports dance movement recognition for human movement monitoring and sensing, including sports dance movement classification algorithm and sports dance movement preprocessing algorithm, which is used to conduct research experiments on sports dance movement recognition for human movement monitoring and sensing. The experimental results of this article show that the average recognition accuracy of the sports dance action recognition system for human motion monitoring and sensing is $92 \%$, which can be used in daily sports dance training and competition.
\end{abstract}

\section{Introduction}

As a new sports project that has evolved and developed in recent centuries, sports dance combines a variety of artistic features, such as clothing, music, dance, and sports. In the existing sports dance training process, dancers usually watch videos repeatedly or seek corrective guidance from coaches to achieve the purpose of training. This not only consumes a lot of time and energy but also brings certain difficulties and challenges to learners.

Under the background of the continuous development of science and technology, human movement-oriented monitoring and sensing technology is gradually applied to sports dance and other fields, so that the majority of dance teaching workers, dancers, and dance learners who have strong needs in teaching and self-study can make accurate adjustments to the dance movements that are not standardized enough to ensure the accuracy of dance positions in training. Trainers can find their own rhythm and sense of rhythm through training and can also promote the further development of digital and basic dance training and other dance learning information.
Gorman et al. studied the relationship between the perception of human actions and the influence of social psychological resources. According to the resource and perception model, it tested and analyzed the common influence of subjective threats and buffer threats to social psychological resources. Two experiments tested whether social threats (i.e., rejection) and psychosocial resources affect the perception of human movement. The observer tries to recognize the movement of the person in the fuzzy point light display after being repelled or not repelled. In addition, the observer tries to measure trait resources (self-esteem plus social support) and manipulate self-affirmation. One of the studies shows that rejection will reduce the sensitivity of detecting human movement, but not for people with rich feature resources. Another study replicated this interaction between rejection phenomena and traits. These studies show that the basic visual skills for detecting human movements are affected by social threats and psychosocial resources. The correlation between the research elements is not strong, and the practicality is weak [1]. Geng et al.'s research found that in the real-time health monitoring, they believe that even 
a subset of available radio frequency functions can provide acceptable classification rates, which can be achieved by making the proposed scheme easier to reduce computational costs. This research is a research on human motion recognition technology for firefighters, and it is not targeted [2]. Chinnadurai and Sridharan found that in recent years, the concept of deep learning has been used to research image processing and machine learning applications. In order to monitor a specific person, Chinnadurai and Sridharan applied a set of image processing steps in the motion area containing the moving object, applied convolutional neural network classifiers with different levels in the selected area, and learned tools in Google TensorFlow used. In the experiment, shaking videos and low-resolution videos were considered and the video dataset was checked. This research process is cumbersome [3].

The innovations of this paper are as follows: (1) propose a classification algorithm using support vector machine model (SVM) as an action recognition method; (2) propose the hardware design of a sports dance action recognition system; and (3) design a sports dance action and identify the software of the system.

\section{Method of Sports Dance Movement Recognition Oriented to Human Motion Monitoring and Sensing}

2.1. Sports Dance Movement Classification Algorithm. This article will use the support vector machine model (SVM) as the classification algorithm of the action recognition method. Support vector machine is a new type of machine learning method based on statistical learning theory and structural risk minimization criteria, and it is also a major achievement of machine learning research in recent years [4]. It finds the global optimal solution from the limited sample information. It not only has the descriptive ability to accurately describe the training sample but also has the learning ability to identify any test sample without error [5]. In this case [6, 7 ], using the $K(x, y)$ function that satisfies the Mercer condition as the inner product operation of the two sample features is equivalent to mapping the sample from the original feature space to a new feature space [8].

The following formula exists $[9,10]$ :

$$
L(W, b, a)=\frac{1}{2}\left(W^{T} \cdot W\right)-\sum_{i=1}^{m} a_{1}\left[a_{i}\left(W^{T} \cdot x_{i}+b\right)-1\right]
$$

$L(W, b, a)$ with respect to $W$ and $b$, and make them equal to zero [11].

$$
\begin{aligned}
\sum_{i=1}^{m} a_{i} y_{i} & =0, \quad a_{i} \geq 0, i=1,2, \cdots, m, \\
W & =\sum_{i=1}^{m} a_{i} y_{i} x_{i},
\end{aligned}
$$

where $a_{i}$ is under the constraint condition $[12,13]$ :

$$
Q(a)=\sum_{i=1}^{m} a_{i}-\frac{1}{2} \sum_{i, j=1}^{m} a_{i} a_{j} y_{i} y_{j}\left(X_{i}^{T} X_{j}\right)
$$

A dual problem has an optimal solution [14]. The solution of this optimization problem must satisfy the following condition:

$$
a_{i}^{*}\left[y_{i}\left(W^{T} \cdot x_{i}+b\right)-1\right]=0, \quad i=1,2, \cdots, m
$$

The model is represented by the number of support vectors [15].

$$
f^{*}(x)=\operatorname{sgn}\left(\sum_{i=1}^{m} a_{i}^{*} y_{i}\left(x_{i} \cdot x\right)+b^{*}\right)
$$

The function $K(x, y)$ from the original feature space to a new feature space [16] was used.

$$
f^{*}(x)=\operatorname{sgn}\left(\sum_{i=1}^{N} a_{i}^{*} y_{i} \cdot K\left(x_{i}, x\right)+b\right) \text {. }
$$

2.2. Sports Dance Movement Preprocessing Algorithm. Sports dance moves contain a lot of noise when recognizing and transforming, and these noises will affect the edge features of dance video images [17]. Therefore, we need to perform preprocessing operations on the dance dataset first [18]. The preprocessing operations in this paper include background subtraction and median filtering operations. Background subtraction is used to extract the foreground to separate the human action area, and the median filtering operation is used to filter out the noise in the image to reduce the impact on edge features $[19,20]$.

2.2.1. Background Subtraction. Regarding background subtraction, the Gaussian mixture model method is adopted, and the video image sequence is regarded as a combination of multiple single Gaussian models. The model maintains a multidistributed density function for each pixel in the image. The video image sequence is usually described as the pixel probability distribution function [21].

Assuming that the value of a certain pixel at time $t$ is $p_{t}$, the probability of occurrence of $p_{t}$ has the following formula:

$$
P\left(p_{t}\right)=\sum_{i=1}^{K} \omega_{i, t} \cdot \eta\left(p_{t}, \mu_{i, t}, \sigma_{i, t}\right)
$$

Among them, $\omega_{i, t}$ is the weight of the $i$ th Gaussian distribution at time $t$, and $\eta\left(p_{t}, \mu_{i, t}, \sigma_{i, t}\right)$ is the corresponding probability density function $[22,23]$. The specific manifestation of $\eta\left(p_{t}, \mu_{i, t}, \sigma_{i, t}\right)$ is

$$
\eta\left(p_{t}, \mu_{i, t}, \sigma_{i, t}\right)=\frac{1}{\sqrt{2 \pi\left|\sigma_{i, t}\right|}} e^{-(1 / 2)\left(p_{t}-\mu_{i, t}\right)^{T} \sigma_{i, t}{ }^{-1}\left(p_{t}-\mu_{i, t}\right)}
$$

And their weights are assigned the same value [24]. 
Assuming that the value of a pixel in a newly input image is $p_{t}$, the following formula can be used to determine whether the pixel matches the established $K$ Gaussian distribution:

$$
\left|p_{t}-\mu_{i, t-1}\right| \leq 2.5 \sigma_{i, t-1} \text {. }
$$

2.2.2. Median Filter. The specific process of median filtering is as follows:

(1) First, move the template in the image, and move the center of the template to coincide with the center pixel of the image [25]

(2) Get the gray value of all pixels under the template

(3) Sort the gray values of all pixels obtained from the template in an ascending order

(4) Calculate the median of all gray values after the arrangement [26]

The specific process is shown in Figure 1.

\section{Experiment of Sports Dance Action Recognition System Oriented to Human Motion Monitoring and Sensing}

\subsection{Hardware Design of Sports Dance Action Recognition System}

3.1.1. System Hardware Architecture. The data acquisition module is used to collect information related to the human body state in real time and provides three-axis acceleration and three-axis angular velocity data for the sports dance action recognition system [27]. It is the data basis of the sports dance action recognition system, so the performance of the entire wearable human body motion state perception system is observed. The pros and cons all depend on the design part of the data acquisition module.

The main composition of the hardware of this system is composed of two parts: data acquisition of sensors and Bluetooth radio frequency. The sensor part is mainly composed of six-axis sensor MPU6050 and air pressure temperature sensor BMP180. The Bluetooth chip uses TI's CC2541. MPU6050 and BMP180 are connected to CC2541 through I2C bus and transmit the collected data to the Bluetooth chip. The Bluetooth chip transmits the collected data through the ceramic antenna on the board.

3.1.2. Motion Sensor Chip MPU6050. The main function of the data acquisition module is to comprehensively collect the acceleration signal and angular velocity signal when the human body moves, so when choosing the sensor chip, give priority to the three-axis accelerometer and the three-axis gyroscope. Based on the above considerations, the sports dance action recognition system designed in this article chooses the MPU6050 motion sensor chip introduced by InvenSense. The MPU6050 motion sensor chip is the world's first integrated 6-axis motion processing chip. It integrates a 3 -axis gyroscope and a 3-axis accelerator into a chip with only a millimeter package. It reduces the size of the space that originally required two motion sensor chips to achieve and greatly reduces the space on the PCB circuit board.

3.1.3. Bluetooth 4.0 Wireless Module. The Bluetooth 4.0 wireless module is composed of the core chip TI's CC2541 and its related circuits.

\subsection{Software Design of Sports Dance Action Recognition System}

3.2.1. C\# Development Language and Its Development Environment. The main content of this paper is to realize the recognition of sports dancers' human movements in a stable environment. Therefore, it is necessary to choose a stable and powerful development environment as the software platform. Due to the powerful functions of Microsoft Visual Studio 2010 and good human-computer interaction, we choose this development environment as the software development platform of this system.

3.2.2. Software Hierarchy and Functions. The human body motion recognition system software for sports dancers designed in this paper adopts a three-layer structure, namely, user layer, business logic layer, and device layer.

The user layer is responsible for displaying the results of the data to users in the form of charts, data, and graphs and accepting user settings and feedback information.

The business logic layer is designed to store, process, and analyze the three-axis acceleration and gyroscope data after receiving the data transmitted by the node.

The device layer is mainly responsible for wireless network interface and communication and works with the upper layer to maintain reasonable and effective network routing and to set the current network topology and protocol.

3.2.3. Using MATLAB's Engine Technology. MATLAB's engine function library is a collection of a series of programs provided by MATLAB, allowing users to call MATLAB in their own $C$ language programs, using MATLAB as a calculation engine, and let it run in the background. Its task is to complete complex matrix operations and simplify the user programming task of the front desk. When the MATLAB engine is started, it is equivalent to starting another process in the background. The MATLAB engine function acts as a bridge between the user application and the MATLAB engine. It completes the exchange of data and the transmission of commands between the two. The three interface functions PutFullMatrixO, ExecuteO, and GetFullMatrix() are mainly used here. These three functions are the three member functions of MLAppClass, so we first need to add the COM application "MatlabApplication (version 7.7) Type Library" to instantiate the engine object, then use its interface for programming. Among the above three functions, PutFullMatrix and GetFullMatrix realize the functions of adding matrix to MATLAB Server and reading matrix from MATLAB Server, and Execute realizes the function of running script.

The specific function form is as follows: matlab. PutFullMatrix("P", "Base”,TrainIn,ImIn); matlab. GetFullMatrix("R", “Base”,TrainOut,ImOut); matlab.

Specific process is shown in Table 1. 


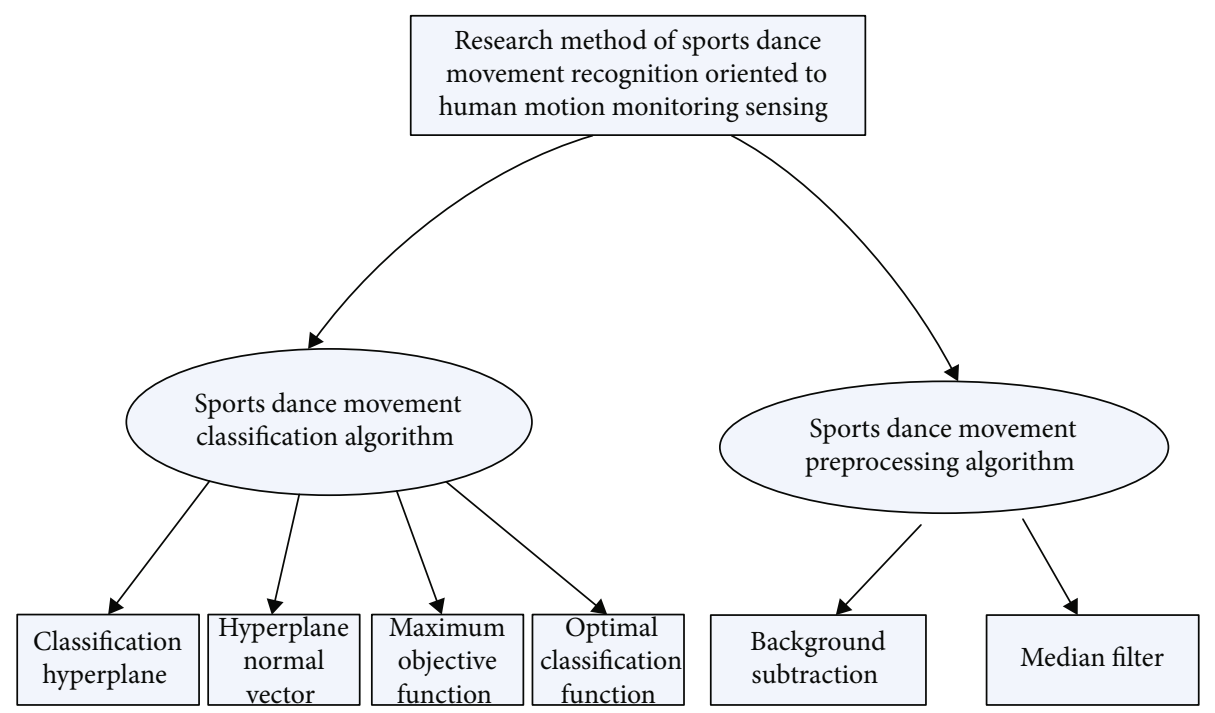

Figure 1: Part of the technical process of this method.

TABLE 1: Experimental steps.

\begin{tabular}{|c|c|c|c|c|}
\hline \multirow{9}{*}{$\begin{array}{l}\text { Research and experiment of sports dance action } \\
\text { recognition system oriented to human motion } \\
\text { monitoring and sensing }\end{array}$} & \multirow{6}{*}{3.1} & \multirow{5}{*}{$\begin{array}{l}\text { Hardware design of sports dance action } \\
\text { recognition system introduction to } \\
\text { identification system }\end{array}$} & 1 & $\begin{array}{c}\text { Introduction to } \\
\text { identification system }\end{array}$ \\
\hline & & & 2 & Power circuit design \\
\hline & & & 3 & Reset circuit design \\
\hline & & & 4 & Clock circuit design \\
\hline & & & 5 & USB circuit design \\
\hline & & \multirow{4}{*}{$\begin{array}{l}\text { Software design of sports dance action } \\
\text { recognition system }\end{array}$} & 6 & $\begin{array}{l}\text { Design of adding meter } \\
\text { interface circuit }\end{array}$ \\
\hline & \multirow{3}{*}{3.2} & & 1 & $\begin{array}{l}\text { C\# development language } \\
\text { and its development } \\
\text { environment }\end{array}$ \\
\hline & & & 2 & $\begin{array}{l}\text { Software hierarchy and } \\
\text { function }\end{array}$ \\
\hline & & & 3 & $\begin{array}{l}\text { Using MATLAB's engine } \\
\text { technology }\end{array}$ \\
\hline
\end{tabular}

\section{Sports Dance Action Recognition for Human Motion Monitoring and Sensing}

\subsection{Preliminary Work Analysis of Action Recognition}

(1) Before performing action recognition on sports dancers, first, classify and analyze the dance movements of sports dancers according to the relevant opinions of professionals. As shown in Table 2 and Figure 2, the average represents the evaluation of the importance of the indicator by professionals, and the coefficient of variation reflects the degree of dispersion of the evaluation of an indicator by professionals

It can be seen from the chart that among the secondary indicators, the coefficients of variation of "crossdance step," "lock step," "flicking," and "inhibition" are all greater than 0.40 . Experts have disputes over these indicators. It is believed that "lock step" steps are mostly locked rotation. It is recom- mended to adjust the name of the index to "lock turn" and move the item to the first-level index "rotation," while doing "crossdance step" and "flick step." The technical link of the index action is not based on mobile technology, but more includes reflexive action position and body rotation. In addition, the first-level index "linear movement" cannot accurately cover the above three second-level index types of actions, so consider adding a level. The indicator "connection category" and the "crossdance step category", "flicking category," and "inhibition category" are included under it, as the secondary indicators of the primary indicator "connection category."

(2) Ask the dancers about their willingness to apply the sports dance action recognition system to the training process, as shown in Table 3 and Figure 3

According to the data, $90.25 \%$ of the dancers are very willing to use the system, $7.75 \%$ do not care whether they use the system or not, and only $2.00 \%$ are reluctant to use 
TABLE 2: Opinion statistics of professionals.

\begin{tabular}{|c|c|c|c|c|c|}
\hline First-level indicator & Secondary indicators & Number & Average & Standard deviation & Coefficient of variation \\
\hline \multirow{4}{*}{ Linear movement } & Transition class & 1 & 3.97 & 0.86 & 0.21 \\
\hline & Rotation & 2 & 4.03 & 0.63 & 0.32 \\
\hline & Screw type & 3 & 4.21 & 0.74 & 0.28 \\
\hline & Axis rotation & 4 & 4.33 & 0.69 & 0.23 \\
\hline \multirow{4}{*}{ Rotating } & Cross dance & 5 & 3.86 & 0.61 & 0.19 \\
\hline & Detours & 6 & 3.65 & 0.56 & 0.31 \\
\hline & Lock step class & 7 & 4.07 & 0.47 & 0.26 \\
\hline & Flutes & 8 & 3.76 & 0.58 & 0.19 \\
\hline \multirow{3}{*}{ Modeling } & Inclined modeling class & 9 & 3.89 & 0.62 & 0.22 \\
\hline & Hinge modeling class & 10 & 4.11 & 0.51 & 0.18 \\
\hline & Sword step modeling & 11 & 4.52 & 0.59 & 0.11 \\
\hline \multirow{4}{*}{ Unique dance steps } & Tango walking class & 12 & 4.28 & 0.38 & 0.14 \\
\hline & Vienna group transfer & 13 & 4.47 & 0.32 & 0.16 \\
\hline & Foxtrot undulate & 14 & 4.16 & 0.44 & 0.25 \\
\hline & Fast step and jump class & 15 & 3.85 & 0.46 & 0.31 \\
\hline
\end{tabular}

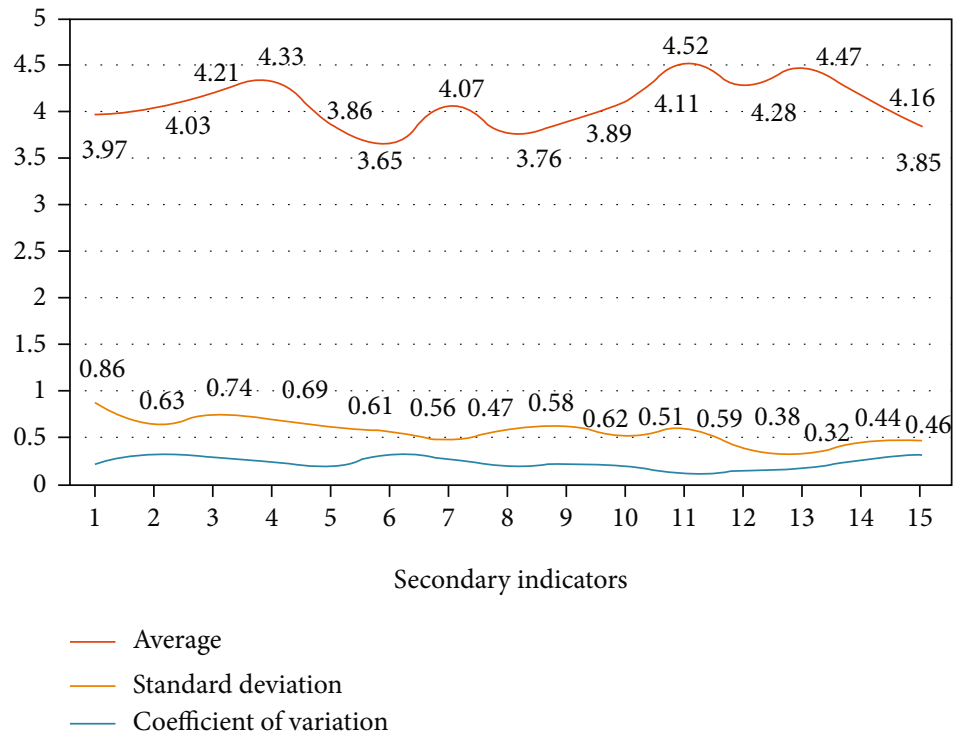

FIgURE 2: Classification of opinions statistics of professionals.

TABLE 3: Related wishes of dancers.

\begin{tabular}{lccc}
\hline & Very willing & Indifferent & Not really \\
\hline Male dancer & $46.14 \%$ & $4.56 \%$ & $1.21 \%$ \\
Female dancer & $44.11 \%$ & $3.19 \%$ & $0.79 \%$ \\
In total & $90.25 \%$ & $7.75 \%$ & $2.00 \%$ \\
\hline
\end{tabular}

the system. Among them, the difference between male and female dancers is small.

\subsection{Performance Analysis of Sports Dance Action Recognition}

(1) Experiment with the algorithm [28] and three features of this paper in the four groups of dance combinations on the FolkDance dance dataset, and count and organize the relevant results, as shown in Table 4 and Figure 4

From the experimental results shown in the chart, it can be seen that the recognition rate of dance movements for each single feature in each group is still relatively low. It can be seen from the table that the action feature extraction method in this paper has the highest recognition and extraction rate for modeling actions and other unique dance steps, which are $55.47 \%$ and $56.19 \%$, respectively; the recognition rate for linear movement dance steps is $49.76 \%$; for rotating dance steps. The recognition extraction rate is $52.32 \%$; the average recognition rate is about $53.44 \%$. 


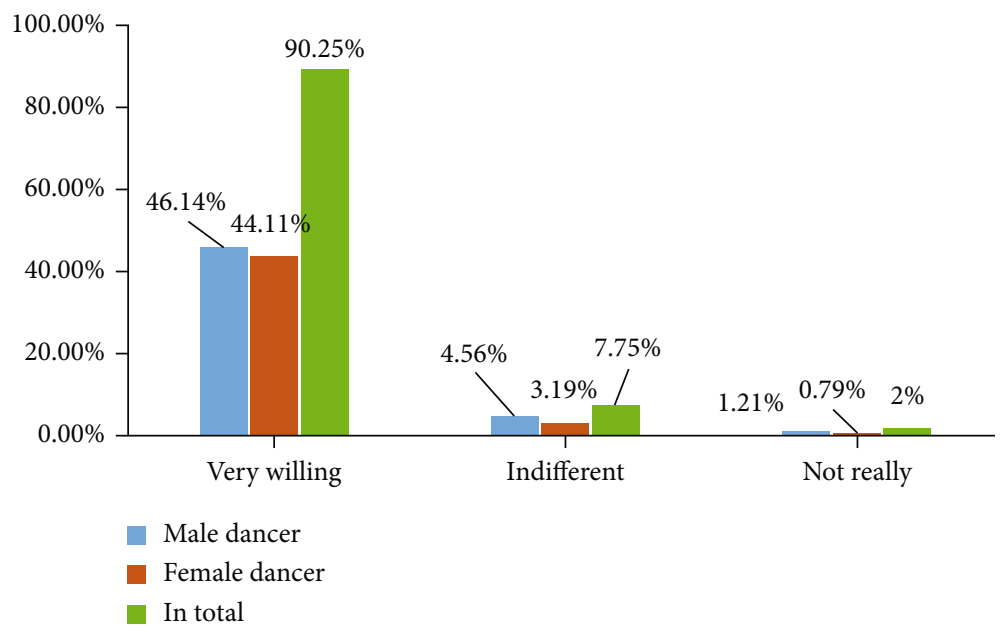

FIgURE 3: The willingness of dancers.

TABLE 4: Comparison of single feature on FolkDance dataset and experimental results of this method.

\begin{tabular}{lcccc}
\hline & $\begin{array}{c}\text { Histogram features of directional } \\
\text { gradient }\end{array}$ & $\begin{array}{c}\text { Histogram characteristics of optical flow } \\
\text { direction }\end{array}$ & $\begin{array}{c}\text { Audio signature } \\
\text { features }\end{array}$ & $\begin{array}{c}\text { The method of this } \\
\text { paper }\end{array}$ \\
\hline $\begin{array}{l}\text { Linear } \\
\text { movement }\end{array}$ & $46.71 \%$ & $40.12 \%$ & $34.61 \%$ & $49.76 \%$ \\
Rotating & $41.23 \%$ & $38.16 \%$ & $39.24 \%$ & $52.32 \%$ \\
Modeling & $39.51 \%$ & $36.44 \%$ & $36.12 \%$ & $55.47 \%$ \\
Unique dance & $36.45 \%$ & $35.43 \%$ & $33.09 \%$ & $56.19 \%$ \\
steps & & & 3 & \\
\hline
\end{tabular}

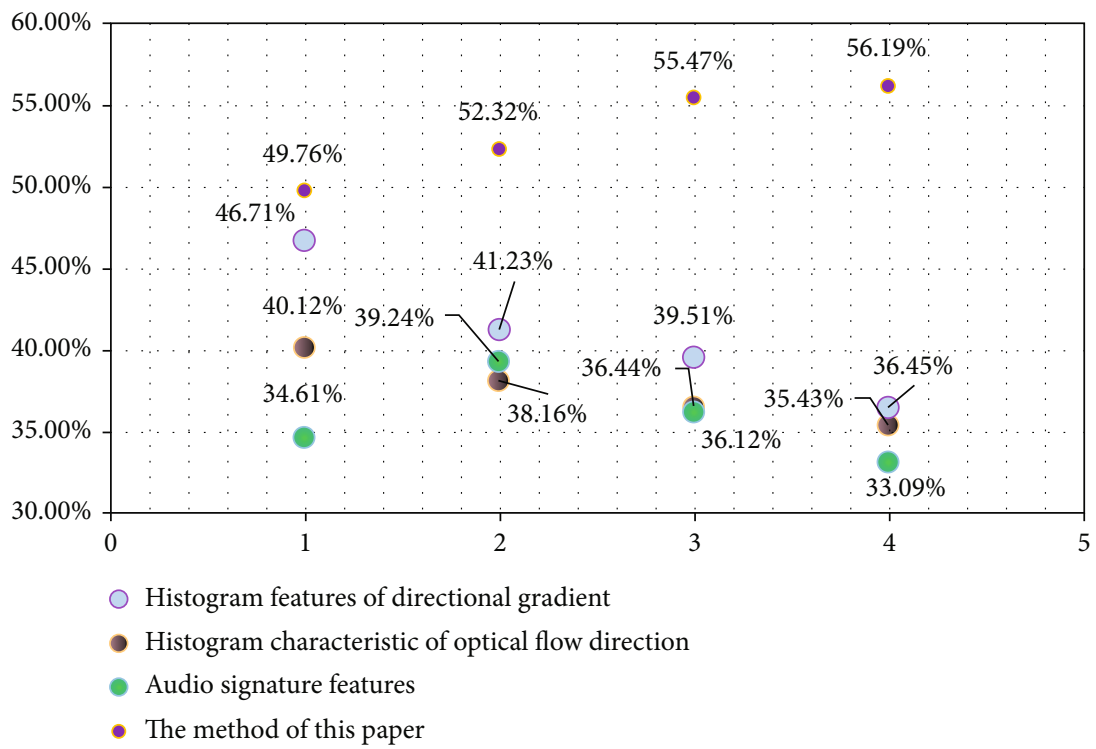

FIgURE 4: Comparison of a single feature on the FolkDance dataset and the experimental results of this method.

(2) In the experiment process, the effect of action recognition in the above database is observed. At the same time, the recognition effect of the combination of 3DSIFT and optical flow is better than the combination of RGB and optical flow. The different feature recognition effects are shown in Table 5 and Figure 5
The recognition rate is the highest when the combination of human body regions is used, and the whole body region is directly recognized.

(3) Sports dance is a dance type with a larger opening and closing of movements, and dancers often fall 
TABLE 5: Different feature recognition effects of different body regions.

\begin{tabular}{|c|c|c|c|c|}
\hline \multirow{2}{*}{ Body parts } & \multicolumn{4}{|c|}{ Northeast Yangko } \\
\hline & RGB & SIFT & Flow & SIFT + flow \\
\hline Upper body & 10.6 & 23.4 & 32.3 & 46.2 \\
\hline Lower body & 11.5 & 26.1 & 35.6 & 45.4 \\
\hline Upper body + lower body & 21.4 & 24.3 & 41.4 & 55.3 \\
\hline Whole body & 22.7 & 22.1 & 40.9 & 53.9 \\
\hline
\end{tabular}

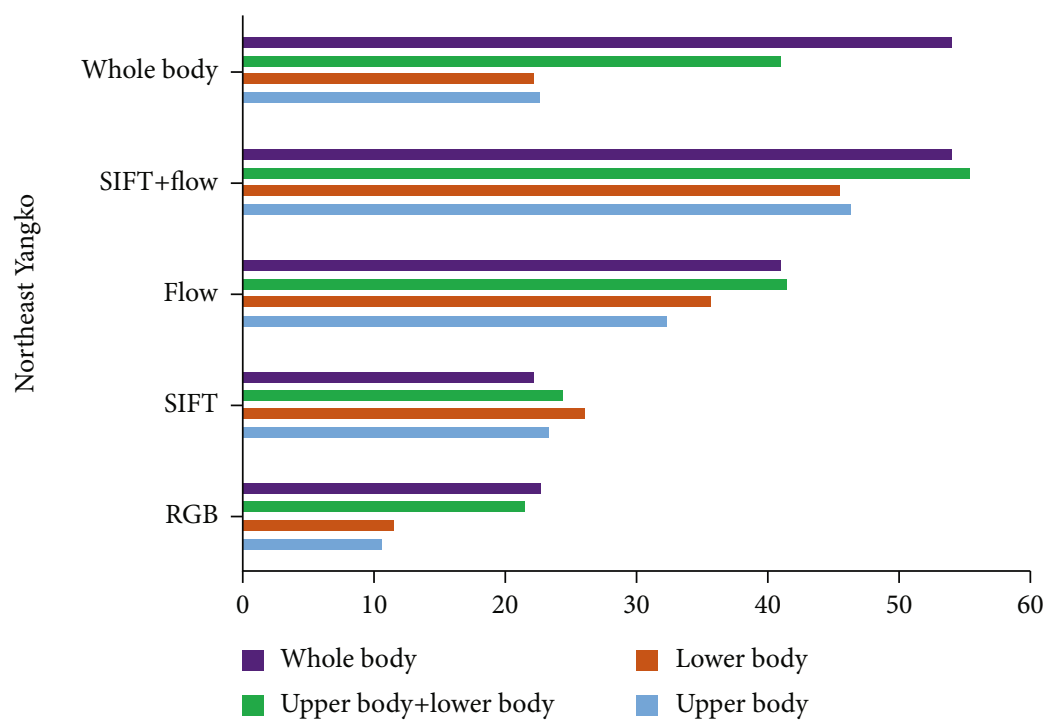

Figure 5: Different feature recognition effects extracted from different body regions.

TABLE 6: Results of different types of falls.

\begin{tabular}{|c|c|c|c|c|c|}
\hline Falls & Total number of experiments & Correct recognition times & Correct rate & Number of false negatives & False-negative rate \\
\hline Fall forward & 25 & 23 & $92 \%$ & 4 & $8 \%$ \\
\hline Fall backward & 25 & 23 & $92 \%$ & 2 & $8 \%$ \\
\hline Fall to the left & 25 & 22 & $88 \%$ & 3 & $12 \%$ \\
\hline Fall to the right & 25 & 24 & $96 \%$ & 1 & $4 \%$ \\
\hline
\end{tabular}

down during training. A protective activation mechanism can be added to the recognition of sports dance movements. The system will automatically send a signal to the team doctor when the dancer falls or has a sports injury, so that the doctor can rush to treat the dancer in time. This article carried out related experiments, and the experimental results recorded the correct identification, underreporting, and false alarming of falls. Among them, correct recognition means correct recognition of the fall and its direction; false negative means that the experimenter did not recognize the fall behavior after the fall. We made a detailed record of the entire experiment process, and the experimental statistical results are shown in Table 6 and Figure 6

It can be seen from the chart that the accuracy of the algorithm proposed in this paper for the recognition of falling behaviors has reached more than $85 \%$, and the average recog- nition accuracy is $92 \%$. There was very little underreporting during the whole experiment.

(4) This paper implements a GM-based recognition method on the segmented action pattern sets of CityU. For each set of experiments, we selected samples collected by 3 of the captured persons as the training set, and the other two samples are used as a test sample. The recognition accuracy rates of GMbased DTW and LCS on the segmented action pattern sets on the CityU and HDM05 datasets are shown in Table 7 and Figure 7

But it has the lowest recognition accuracy on the HDM05 dataset. This is because the HDM05 dataset has more significant time domains than the CityU dataset, through its own penalty mechanism, unlike DTW, which uses repeated E matching for longer samples in the middle frame, which is extremely easily brings overfitting problems. 


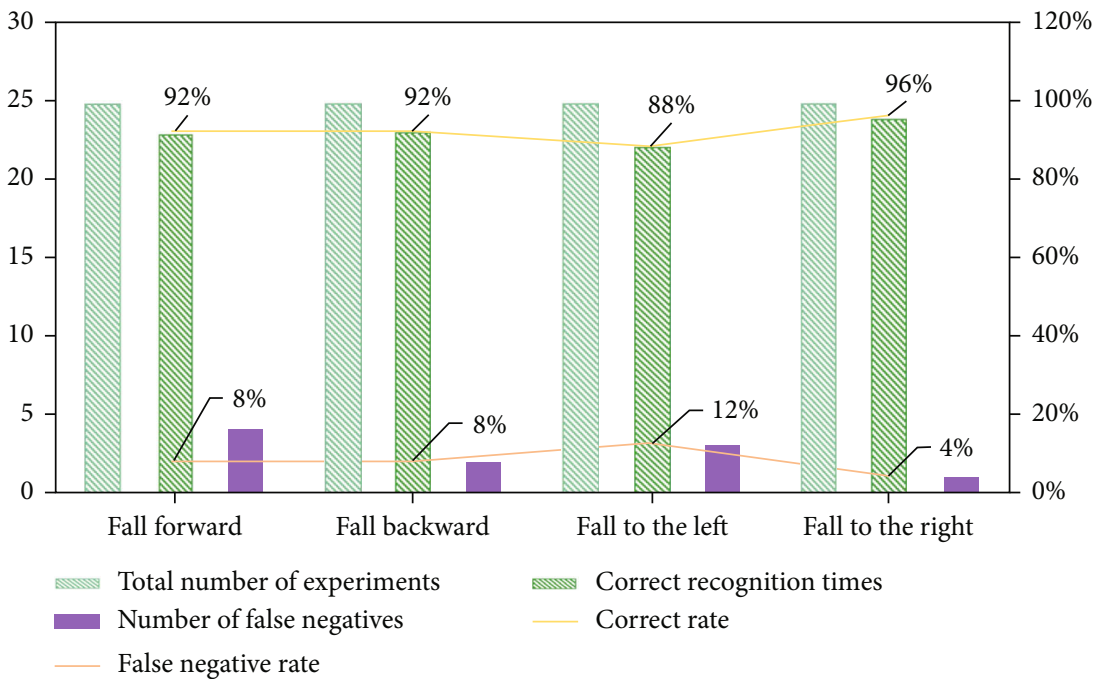

Figure 6: Results of different types of falls.

TABLE 7: Recognition accuracy based on GM.

\begin{tabular}{lclll}
\hline & & Team 1 & Team 2 & Team 3 \\
\hline \multirow{2}{*}{ CityU } & DTW & $93.26 \%$ & $93.17 \%$ & $93.21 \%$ \\
& LCS & $91.12 \%$ & $90.19 \%$ & $91.07 \%$ \\
\multirow{2}{*}{ HDM05 } & DTW & $95.34 \%$ & $88.49 \%$ & $89.14 \%$ \\
& LCS & $97.47 \%$ & $86.23 \%$ & $86.24 \%$ \\
\hline
\end{tabular}

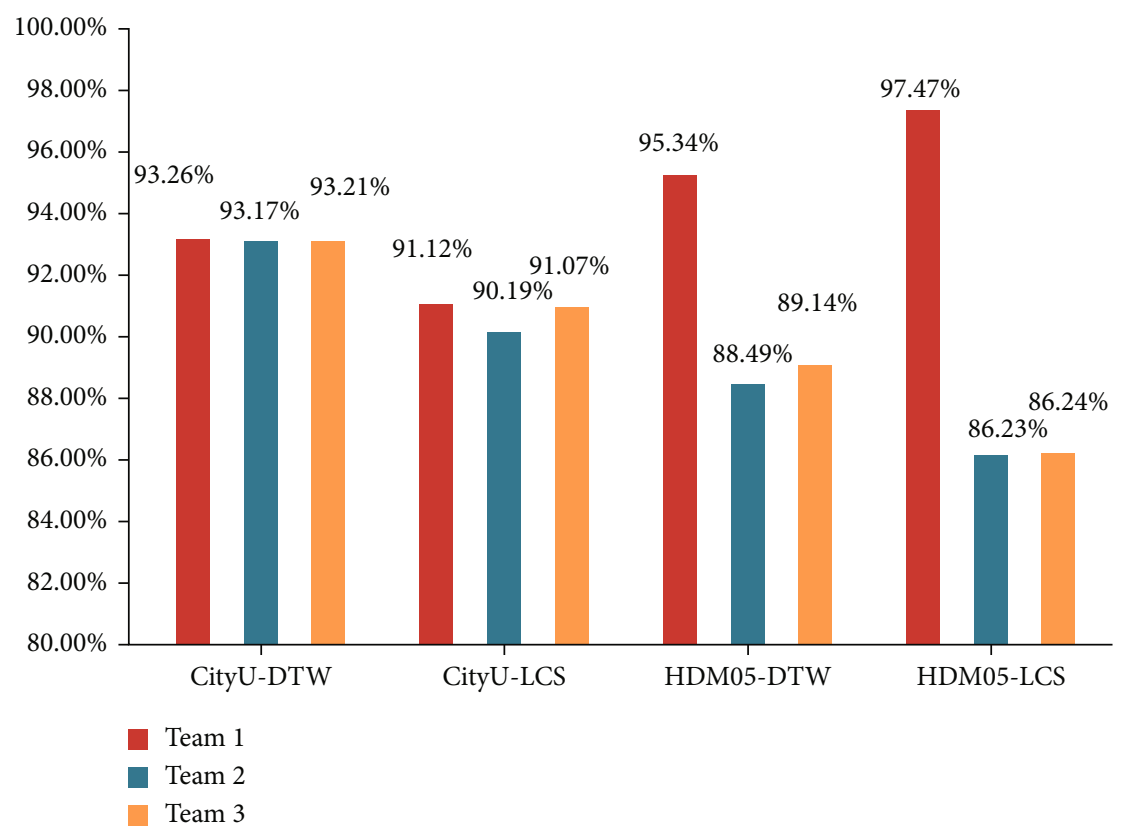

FIgURE 7: Recognition accuracy based on GM.

\section{Conclusions}

Sports dance is a sport which includes standard dance and Latin dance. It has gone through three stages: folk dance, standard dance, and competitive dance. Less than a century ago, it has triggered a global upsurge of sports dance and competitive sports dance. In the 1930s, it was introduced into China in the form of social dance. At present, four international dance sports organizations, including the world sports dance federation, the World Dance Federation, the Royal 
Association of dance teachers, and the International Association of dance teachers, are committed to the development and popularization of dance sport in the world.

Motion capture technology is used to track the motion of some or all joints of the human body or animal through video devices, motion sensors, and other devices and measure the motion information of joints, so as to provide reference data for gait recognition and video production. This technology has been widely used in film and television production, interactive games, virtual reality, and personnel training. In order to better correct the movement of sports dancers and to have accurate training, the motion capture recognition technology is introduced into the design of sports dance action recognition system.

This paper designs the hardware part and software part of the system. The software part includes data acquisition, data processing, and auxiliary training. In the data acquisition part, the standard dance movements are input into the system, and the standard human dance posture database is established. The data processing part repairs the occluded joint points and restores the human skeleton structure. The auxiliary training part is divided into two parts, which are based on the joint point position and the joint angle. The system has the effect of assisting training. It mainly provides real suggestions for the trainer's training through data analysis, and the suggester can make reasonable adjustments based on the suggestions. The experimental results show that the system can effectively improve the dance level of trainers.

\section{Data Availability}

No data were used to support the study.

\section{Disclosure}

We confirm that the content of the manuscript has not been published or submitted for publication elsewhere.

\section{Conflicts of Interest}

There are no potential competing interests in our paper.

\section{Authors' Contributions}

All authors have seen the manuscript and approved its submission.

\section{Acknowledgments}

This work was supported by the Heilongjiang provincial basic scientific research business expenses in school undergraduate course colleges and universities cooperation projects (135409510) and Heilongjiang provincial undergraduate college basic scientific research business expenses project (Heilongjiang and brand cultivation of ice and snow sports cultural industry development stage results).

\section{References}

[1] J. L. Gorman, K. D. Harber, M. Shiffrar, and K. S. Quigley, "Ostracism, resources, and the perception of human motion," European Journal of Social Psychology, vol. 47, no. 1, pp. 53-71, 2017.

[2] Y. Geng, J. Chen, R. Fu, G. Bao, and K. Pahlavan, "Enlighten wearable physiological monitoring systems: on-body RF characteristics based human motion classification using a support vector machine," IEEE Transactions on Mobile Computing, vol. 15, no. 3, pp. 656-671, 2016.

[3] M. Chinnadurai and M. Sridharan, "Prediction of human motion detection in video surveillance environment using tensor flow," International Journal of Advanced Science and Technology, vol. 29, no. 5, pp. 2791-2798, 2020.

[4] M. Sun, Y. Jiang, Q. Liu, and X. Liu, "An auto-calibration approach to robust and secure usage of accelerometers for human motion analysis in FES therapies," Computers, Materials \& Continua, vol. 60, no. 1, pp. 67-83, 2019.

[5] B. Ruscello, M. Esposito, G. Siligato et al., "Gender differences in instep soccer kicking biomechanics, investigated through a 3D human motion tracker system," The Journal of Sports Medicine and Physical Fitness, vol. 60, no. 8, pp. 1072-1080, 2020.

[6] J. Lin and X. Huang, "Application of clothing friction nanopower generators in human motion energy acquisition," Nanomaterials and Energy, vol. 9, no. 2, pp. 163-172, 2020.

[7] H. Mai, R. Mutlu, C. Tawk, G. Alici, and V. Sencadas, "Ultrastretchable MWCNT-Ecoflex piezoresistive sensors for human motion detection applications," Composites Science and Technology, vol. 173, no. MAR.22, pp. 118-124, 2019.

[8] X. Zhou, M. Zhu, G. Pavlakos, S. Leonardos, K. G. Derpanis, and K. Daniilidis, "MonoCap: monocular human motion capture using a CNN coupled with a geometric prior," IEEE Transactions on Pattern Analysis and Machine Intelligence, vol. 41, no. 4, pp. 901-914, 2019.

[9] S. Park, H. Ryu, S. Lee, S. Lee, and J. Lee, "Learning predictand-simulate policies from unorganized human motion data," ACM Transactions on Graphics, vol. 38, no. 6, pp. 1-11, 2019.

[10] W. Mrabti, H. Tairi, F. M. Nicolier, and B. Bellach, "Human motion tracking under indoor and outdoor surveillance system," International Journal of Innovative Computing and Applications, vol. 11, no. 4, pp. 181-192, 2020.

[11] J. S. Park, C. Park, and D. Manocha, "I-Planner: intentionaware motion planning using learning-based human motion prediction," The International Journal of Robotics Research, vol. 38, no. 1, pp. 23-39, 2019.

[12] P. B. Zhang and Y. S. Hung, "Articulated deformable structure approach to human motion segmentation and shape recovery from an image sequence," IET Computer Vision, vol. 13, no. 3, pp. 267-276, 2019.

[13] M. M. Rana and R. Bo, "IoT-based improved human motion estimations method under cyber attacks," IEEE Internet of Things Journal, vol. 6, no. 6, pp. 10934-10935, 2019.

[14] C. K. Chan and G. Cala, "Impact of number of attributes on the accuracy of human motion classification," Journal of Engineering Science and Technology, vol. 13, no. 7, pp. 1967-1978, 2018.

[15] Y. Lang, Q. Wang, Y. Yang, C. Hou, D. Huang, and W. Xiang, "Unsupervised domain adaptation for micro-Doppler human motion classification via feature fusion," IEEE Geoscience and Remote Sensing Letters, vol. 16, no. 3, pp. 392-396, 2019. 
[16] P. Wang, H. Liu, L. Wang, and R. X. Gao, "Deep learning-based human motion recognition for predictive context-aware human-robot collaboration," CIRP Annals - Manufacturing Technology, vol. 67, no. 1, pp. 17-20, 2018.

[17] M. C. Sashikkumar, S. Selvam, N. Karthikeyan, J. Ramanamurthy, S. Venkatramanan, and C. Singaraja, "Remote sensing for recognition and monitoring of vegetation affected by soil properties," Journal of the Geological Society of India, vol. 90, no. 5, pp. 609-615, 2017.

[18] W. Kumne and S. Samanta, "Remote sensing and GIS application on forest resource mapping and monitoring in Bulolo district, Morobe province," Journal of Geoscience and Environment Protection, vol. 7, no. 2, pp. 37-48, 2019.

[19] G. Xia, H. Sun, B. Chen et al., "Nonlinear low-rank matrix completion for human motion recovery," IEEE Transactions on Image Processing, vol. 27, no. 6, pp. 3011-3024, 2018.

[20] L. Davaze, A. Rabatel, Y. Arnaud et al., "Monitoring glacier albedo as a proxy to derive summer and annual surface mass balances from optical remote-sensing data," The Cryosphere, vol. 12, no. 1, pp. 271-286, 2018.

[21] R. Li, Z. Liu, and J. Tan, "Human motion segmentation using collaborative representations of $3 \mathrm{D}$ skeletal sequences," IET Computer Vision, vol. 12, no. 4, pp. 434-442, 2018.

[22] D. H. Yoo, "Effects of dance sports and yoga program on body composition, physical fitness, blood lipids and liver function Indicator in the elderly," Exercise Science, vol. 29, no. 1, pp. 51-59, 2020.

[23] M. O. Kim, Y. S. Kim, S. W. Chun, and S. K. Shin, "Effects of low intensity dance sports exercise for 16 weeks on senior fitness test and Fullerton advanced balance in elderly man," The Korean Journal of Growth and Development, vol. 27, no. 4, pp. 321-327, 2019.

[24] Wookyungkim and C. Park, "Casual relation between the partner confidence, exercise immersion and performance ability in the college dance sports athletes," The Journal of Korean Alliance of Martial Arts, vol. 21, no. 1, pp. 89-99, 2019.

[25] I. Lee and J. S. Ham, "Role performance difficulties and overcome strategies experienced by dance sports female instructors," Journal of Korean Society for the Study of Physical Education, vol. 23, no. 3, pp. 175-190, 2018.

[26] R. Xuebing, "81.Application of data mining for Investigating the cognition of how square dance promote community sports culture construction," Boletin Tecnico/technical Bulletin, vol. 55, no. 13, pp. 594-600, 2017.

[27] L. Chen, H. Sun, W. Zhao, and T. Yu, "Robotic arm control system based on AI wearable acceleration sensor," Mathematical Problems in Engineering, vol. 2021, Article ID 5544375, 13 pages, 2021.

[28] Z. Liqiu, Z. Yuexi, and W. Xiaodong, "Athlete's physical fitness prediction model algorithm and index optimization analysis under the environment of AI," Mathematical Problems in Engineering, vol. 2021, Article ID 6680629, 10 pages, 2021. 